\title{
miR-103a-3p alleviates oxidative stress, apoptosis, and immune disorder in oxygen-glucose deprivation-treated BV2 microglial cells and rats with cerebral ischemia-reperfusion injury by targeting high mobility group box 1
}

\author{
Jianshe $\mathrm{Li}^{1}$, Wenlong $\mathrm{He}^{1}$, Yan Wang ${ }^{2}$, Jianting Zhao ${ }^{1}$, Xinli Zhao ${ }^{3}$ \\ ${ }^{1}$ Department of Neurology, Xinxiang Central Hospital, Xinxiang, China; ${ }^{2}$ Department of Neurology, The Third Affiliated Hospital of Xinxiang \\ Medical College, Xinxiang, China; ${ }^{3}$ Department of Neurosurgery, The First Affiliated Hospital of Xinxiang Medical College, Xinxiang, China \\ Contributions: (I) Conception and design: J Li, X Zhao; (II) Administrative support: X Zhao; (III) Provision of study materials or patients: W He, Y \\ Wang; (IV) Collection and assembly of data: J Zhao, J Li; (V) Data analysis and interpretation: J Li, X Zhao; (VI) Manuscript writing: All authors; (VII) \\ Final approval of manuscript: All authors. \\ Correspondence to: Xinli Zhao. Department of Neurosurgery, The First Affiliated Hospital of Xinxiang Medical College, Xinxiang 453000, China. \\ Email: ruiduanren912@163.com.
}

Background: Cerebral ischemia-reperfusion injury (CI/R) is among the most common diseases affecting the central nervous system. Due to the poor efficacy and adverse side effects of the drugs used to treat CI/R in clinical trials, a new treatment strategy is urgently needed. In this study, we aimed to investigate whether miR-103a-3p alleviates CI/R in vivo and vitro and to explore the relevant mechanisms.

Methods: BV2 microglial cells underwent oxygen-glucose deprivation (OGD) treatment to imitate the pathophysiology of CI/R in vitro. A middle cerebral artery occlusion (MCAO) rat model was established to imitate the pathophysiology of CI/R in vivo. The expression levels of miR-103a-3p and HMGB1 were detected by reverse transcription-polymerase chain reaction (RT-PCR) and western blot. Flow cytometry, terminal deoxynucleotidyl transferase dUTP nick end labeling (TUNEL) assay, enzyme-linked immunosorbent assay (ELISA), and hematoxylin and eosin (H\&E) and Nissl staining were used to evaluated apoptosis, oxidative stress, inflammatory response, and histopathology, respectively.

Results: OGD-stimulated BV2 microglial cells and brain tissues with CI/R had low expression of miR$103 \mathrm{a}-3 \mathrm{p}$ but high expression of high mobility group box 1 (HMGB1). As expected, miR-103a-3p and HMGB1 had a targeting relationship. Overexpression of HMGB1 enhanced the the levels of interleukin (IL)-1 beta, tumor necrosis factor-alpha (TNF- $\alpha$ ) and malondialdehyde (MDA), but reduced the content of superoxide dismutase (SOD), IL-4, and IL-10, in vitro. Moreover, high expression of HMGB1 aggravated the brain injury of the model rats, and increased the secretion of inflammatory factors, exacerbated oxidative stress, and further induced tissue apoptosis in the brain tissue. Importantly, these effects of HMGB1 overexpression were partly reversed by miR-103a-3p overexpression on HMGB1 interference.

Conclusions: HMGB1 is targeted by miR-103a-3p, which may be a new strategy in the treatment of CI/R.

Keywords: BV2 microglial cells; mirRNA; cerebral indexes; adult male Sprague-Dawley rats (SD rats); cerebral ischemia-reperfusion injury (CI/R)

Submitted Jul 03, 2020. Accepted for publication Oct 11, 2020.

doi: $10.21037 / \mathrm{atm}-20-5856$

View this article at: http://dx.doi.org/10.21037/atm-20-5856 


\section{Introduction}

Cerebral ischemia is one of the most common diseases of the central nervous system (1). Reperfusion of ischemic brain tissues can help to avoid severe neurological impairment and death; however, over reperfusion can further exacerbate the neurological deficit and neuronal death (2). Adverse reactions caused by reperfusion injury involve multiple pathophysiological mechanisms, including mitochondrial injury, oxidative stress, elevated intracellular $\mathrm{Ca} 2+$, activation of the inflammatory response, and failure of metabolic energy $(3,4)$. The drugs and therapies used to treat cerebral ischemia-reperfusion injury $(\mathrm{CI} / \mathrm{R})$ in clinical trials have shown adverse side effects and poor efficacy. Therefore, a novel treatment strategy is urgently needed.

MicroRNAs (miRNAs) are endogenous, noncoding RNAs of approximately 22 nucleotides in length that regulate gene expression by combining with the 3 '-untranslated region (3'-UTR) of miRNAs to suppress translation or induction of miRNA cleavage (5). A large body of research suggests that miRNAs regulate various aspects of brain diseases, including neurodegenerative diseases, neuropsychiatric diseases, and cancer $(6,7)$. miRNAs such as miR-200 family, miRNA-145, miRNA-338, miRNA-181, miR-21, and miR-155 were recently reported to be involved in CI/R (8). Recent studies have shown that miRNA-103 performs different physiological functions in multiple diseases, including regulating oxidative stress (9), inhibiting apoptosis $(10,11)$ and alleviating inflammation (12). In cerebral ischemia, Kang et al. demonstrated that upregulation of miR-103 exerts neuroprotective effects (13).

High mobility group box 1 (HMGB1), a universal nonhistone DNA binding protein, is a common inflammatory regulator (14). The imbalance of HMGB1 can result in the development of various inflammatory diseases (14). HMGB1 sensitizes multiple signaling pathways associated with inflammation, which leads to the production of proinflammatory cytokines (15-17). The regulation of HMGB1 is reported to be closely related to CI/R. Yan et al. reported that glycyrrhizic acid exerted a protective effect on CI/R via the suppression of the HMGB1-

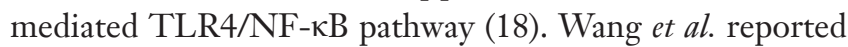
HMGB1 inhibition alleviated CI/R in diabetic mice (19). Li et al. reported that upregulation of miR-129-5p improved neuroinflammation and blood spinal cord barrier injury after ischemia reperfusion through suppressing the TLR3 cytokine pathway and HMGB1 (20).

Although a number of miRNAs are involved in CI/R; however, whether miR-103a-3p can improve the adverse reactions induced by $\mathrm{CI} / \mathrm{R}$ via HMGB1 regulation has yet to be fully established. In this study, we aimed to investigate the effect of miR-103a-3p on CI/R in vitro and in vivo. Concurrently, we explored the relationship between the effect of miR-103a-3p on CI/R and HMGB1 regulation. We present the following article in accordance with the ARRIVE reporting checklist (available at http://dx.doi. org/10.21037/atm-20-5856).

\section{Methods}

\section{Chemicals and reagents}

BV2 microglial cells (ATCC; Manassas, VA, USA) were cultured in Dulbecco's Modified Eagle Medium (DMEM, Thermo, Waltham, MA, USA) with $0.25 \%$ trypsin and $5 \%$ fetal bovine serum (FBS) in a $5 \% \mathrm{CO}_{2}$ incubator. A BCA protein assay kit was purchased from Pierce (Thermo, Waltham, MA, USA). Primary antibodies were obtained from Cell Signaling Technology (Danvers, MA, USA). The small interfering RNA (siRNA) targeting HMGB1, miR103a-3p mimic, anti-miR-103a-3p, HMGB1 (ORF), and vector were bought from GenePharma (Shanghai, China). The horseradish peroxidase (HRP)-conjugated rabbit antirat IgG secondary antibodies were supplied by BIOSS (Beijing, China). The kits for the detection of inflammatory factors were obtained from Shanghai Qiaoyu Biotechnology Co., Ltd. (Shanghai, China).

\section{OGD/R injury}

To simulate I/R conditions in vitro, BV2 microglia cells were treated with OGD/R according to methods previously described, with minor adjustments $(21,22)$. Briefly, the cells were washed twice with glucose-free Earle's Balanced Salt Solution and stored in glucose-free DMEM without FBS. Next, the cells were transferred into an anaerobic chamber containing $95 \% \mathrm{~N}_{2}$ and $5 \% \mathrm{CO}_{2}$, and subsequently placed at $37{ }^{\circ} \mathrm{C}$ for 4 hours. Then, the cells were returned to the conventional medium and cultured in an ordinary incubator for a further 24 hours. The control cells were cultured continuously under normal conditions without exposure to OGD/R.

\section{Animals model}

All animal experiments were approved by the Ethics Committee of the Institutional Animal Care and Use Committee of Xinxiang Central Hospital (Henan, China) 
(No. XXZXYY-2019093) and conducted in line with the Guide for the Care and Use of Laboratory Animals (2011).

Adult male Sprague-Dawley (SD) rats were purchased from Vital River Laboratory Animal Technology Co., Ltd. The rats were housed under controlled conditions $\left(23 \pm 2^{\circ} \mathrm{C}\right.$, $60 \% \pm 10 \%$ humidity; light cycle 8 a.m. to 8 p.m.), with free access to food and water.

Thirty-six adult male SD rats underwent focal ischemia using the MCAO method. Briefly, the rats were anesthetized with pentobarbital $(60 \mathrm{mg} / \mathrm{kg}$ i.p.). The body temperature of the rats was kept at $37 \pm 0.5^{\circ} \mathrm{C}$ using a temperature controlled heating pad (CMA 150 Carnegie Medicin, Sweden). The right common carotid artery (CCA), external carotid artery (ECA), and internal carotid artery (ICA) were carefully exposed. The middle cerebral artery was blocked by inserting a 4-0 surgical nylon monofilament (diameter, $0.24 \mathrm{~mm}$ ) into the ICA via the right ECA. After 2 hours of MCAO, the occluded fibers were removed to allow reperfusion. The ECA was tightened and the skin was closed to complete the procedure. The sham group rats underwent the same surgical procedure but excluding MCAO.

The rats were randomly assigned into 4 groups: the sham group ( $\mathrm{n}=6)$, the CI/R group ( $\mathrm{n}=10$, injected with normal saline), the CI/R + miR-103a-3p NC group ( $\mathrm{n}=10$, injected with $50 \mathrm{nM}$ miR-103a-3p NC), and the CI/R + miR-103a$3 \mathrm{p}$ mimics group $(\mathrm{n}=10$, injected with $50 \mathrm{nM}$ miR-103a$3 \mathrm{p}$ mimics). The rats in sham group were injected with normal saline only through the femoral vein. All drugs were administered at $10 \mathrm{~mL} / \mathrm{kg}$ once daily for 3 consecutive days before MCAO. $1.5 \mathrm{mg} / \mathrm{kg}$ Nifedipine was used as positive control in animal experiments. The dose selection is based on previous literature (23).

\section{Histology}

Brain injury in each group of rats was detected by hematoxylin and eosin (H\&E) and Nissl staining.

$\mathrm{H} \& \mathrm{E}$ staining: brain tissue collected from the rats was fixed in 4\% paraformaldehyde for 24 hours and embedded in paraffin. Then, the tissue was sliced into $4-\mu \mathrm{m}$ sections. The slices were stained with $\mathrm{H} \& \mathrm{E}$ and toluidine blue, and then the pathological changes of the tissues were observed under light microscopy. Five random visual fields were selected for each section.

Nissl staining: Nissl staining was carried out using a previously described method with slight modifications (24). After drying, the unstained slides were put into a series of solutions for Nissl staining. Permount (Fisher, Fairlawn,
NJ, USA) was used to cover the slides.

\section{Enzyme-linked immunosorbent assay (ELISA)}

The levels of interleukin 1 beta (IL-1 $\beta$ ), interleukin- 4 (IL-4), tumor necrosis factor-alpha (TNF- $\alpha$ ), and interleukin-10 (IL-10) in OGD-treated BV2 cells and rat brain tissue were determined using ELISA kits, in line with the manufacturer's protocol. The contents of malondialdehyde (MDA) and superoxide dismutase (SOD) were measured using kits, strictly according to the instructions of the manufacturer.

\section{Terminal deoxynucleotidyl transferase dUTP nick end labeling (TUNEL) assay}

After being dewaxed and rehydrated, the sections were washed and then incubated with TUNEL reaction mixture (Roche Diagnostics, Indianapolis, IN, USA) according to the instructions of the manufacturer. Apoptosis in the tissue sections was detected by fluorescence microscopy (Olympus, Japan).

\section{Flow cytometry}

Cell apoptosis was measured by flow cytometry, as described previously (25). Annexin V-FITC (fluorescein isothiocyanate) staining was used to detect apoptotic cells, and cell death was evaluated using the propidium iodide (PI) exclusion method. Finally, a flow cytometry FACSCalibur instrument (BD Biosciences) was used for analysis.

\section{Real-time polymerase chain reaction (PCR)}

HMGB1 was extracted from cells and tissues with TRIzol reagent (Invitrogen, USA).

Reverse transcription of the extracted protein was performed using a reverse transcription-polymerase chain reaction (RT-PCR) kit (TaKaRa, Japan). Real-time PCR was carried out using SYBRExScript ${ }^{\mathrm{TM}}$ RT-PCR Kit (TaKaRa, Japan) on an iQ5 Multicolor Real-Time PCR Detection System (Bio-Rad, Hercules, CA, USA) following the manufacturer's protocol. The relative expression of HMGB1 was analyzed by the comparative $2^{-\triangle \triangle \mathrm{CT}}$ method.

\section{Luciferase reporter assay}

TargetScan software showed that HMGB1 and miR-103a- 
$3 p$ have an obvious targeting relationship. The wild-type (wt) fragments from HMGB1 (HMGB1-wt) with the potential binding sites, mutant type (mut) fragments from HMGB1 (HMGB1-mut), and miR-103a-3p were inserted into the pMIR-report plasmid vector (Promega, USA) to generate the HMGB1 luciferase reporter construct. Then, the HMGB1 mimics and vectors were co-transfected into BV2 microglial cells using Lipofectamine 2000 reagent (Invitrogen, Carlsbad, CA, USA). After 24 hours of transfection, the cells were detected using a luciferase detection kit (Promega, USA), and the ratio of Rluc/Luc was recorded.

\section{Evaluation of neurological deficit}

After $24 \mathrm{~h}$ of reperfusion, the neurological deficit of the rats was scored by an investigator blinded to the study protocol according to the 5-point scoring system as follows (26): (I) 0 : the rat can walk normally or without neurologic deficit; (II) 1: the rat cannot extend their contralateral forepaw fully or displays a mild focal neurologic deficit; (III) 2: the rat exhibits circling to the contralateral side or a moderate focal neurologic deficit; (IV) 3: the rat falls to the contralateral side or shows a severe focal neurologic deficit; (V) 4: the rat cannot walk spontaneously and shows a depressed level of consciousness.

\section{Measurements of brain edema}

Brain edema was measured with the standard wet-dry method. The brains of the rats were removed at 24 hours after reperfusion. The two hemispheres were weighed (wet weight) separately and were dried at $100{ }^{\circ} \mathrm{C}$ for 24 hours to measure the dry weight.

\section{Western blot}

Brain tissue and OGD-stimulated BV2 microglial cells were processed for western blot as previous described (27). Brain tissues $(0.1 \mathrm{~g})$ taken from the ipsilateral side of each group of rats were homogenized in $1 \mathrm{~mL}$ of protein extraction buffer. Then, the tissues were centrifuged, the supernatant was collected, and the protein was detected with a BCA assay kit. Samples of total protein $(20 \mu \mathrm{g})$ were put into $10 \%$ sodium dodecyl sulfate polyacrylamide gel electrophoresis (SDS PAGE) loading buffer and then transferred to polyvinylidene difluoride (PVDF) membranes. The membranes were sealed with $5 \%$ skimmed milk for 120 minutes at $37{ }^{\circ} \mathrm{C}$. Then, the membranes were incubated with the following primary antibodies overnight at $4{ }^{\circ} \mathrm{C}$ : MHGB1 (ab18256, 1:500, Abcam, UK). Then, the membranes and goat anti rabbit IgG HRP were co- incubated at $37^{\circ} \mathrm{C}$ for 60 minutes. The protein band densities were measured and analyzed with BioRad CFX-96 (Bio-Rad, CA, USA).

\section{Statistical analysis}

The statistical analysis was conducted with SPSS 21.0 (SPSS, Inc., Chicago, IL, USA). All experiments were repeated at least three times. Data were presented as the mean \pm SD. Multiple sets of data were analyzed by one-way analysis of variance followed by the Bonferroni post-hoc test.

\section{Results}

\section{Influence of miR-103a-3p interference on HMGB1 expression in OGD-treated BV2 cells}

First, the effects of OGD treatment for different lengths of time $(0,3,6$, and 12 hours) on the expression levels of miR103a-3p and HMGB1 in BV2 cells were studied. As shown in Figure $1 A, B$, the longer the cells were exposed to OGD, the lower the expression of miR-103a-3p was, while the expression of HMGB1 became significantly up-regulated with time compared with the control group. Then, miR$103 a-3 p$ mimics and anti- miR-103a-3p were constructed (Figure 1C). The results showed that overexpression of miR103a-3p inhibited HMGB1 expression, whereas inhibition of miR-103a-3p expression had the opposite effect (Figure 1D,E,F,G). We further verified the relationship between miR-103a-3p expression and HMGB1 expression in OGD-treated BV2 cells. As shown in Figure 1H,I, f,K, miR-103a-3p mimic inhibited HMGB1 expression, while anti-miR-103a-3p promoted HMGB1 expression compared with the miR-103a-3p NC group, at both the relative RNA level and the relative protein level.

\section{miR-103a-3p alleviates LPS-induced oxidative stress and apoptosis in BV2 glial cells by targeting HMGB1}

TargetScan search software showed that miR-103a-3p can bind directly to the 3'-UTR region of HMGB1 (Figure 2A). The results of double luciferase reporter assay showed that miR-103a-3p could significantly suppress the fluorescence intensity of HMGB1 3'-UTR-wt vector $(\mathrm{P}<0.01)$; however, it could not suppress the fluorescence intensity 
A

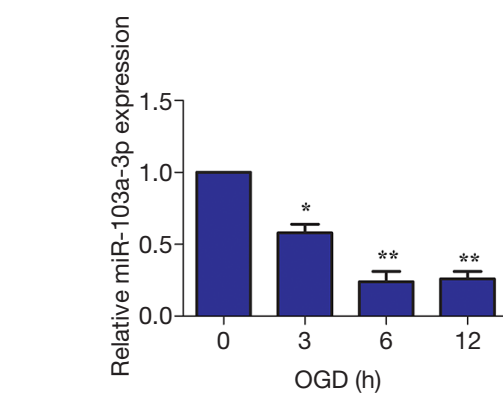

C

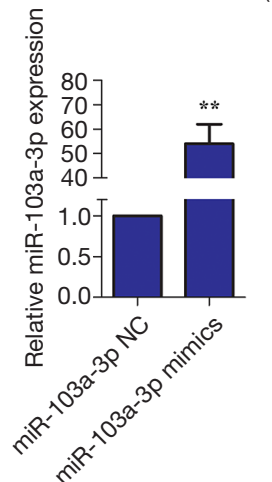

$\mathrm{F}$

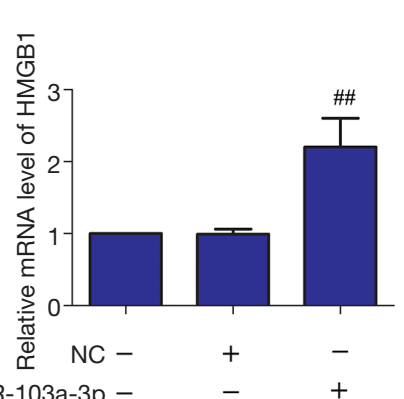
anti-miR-103a-3p -

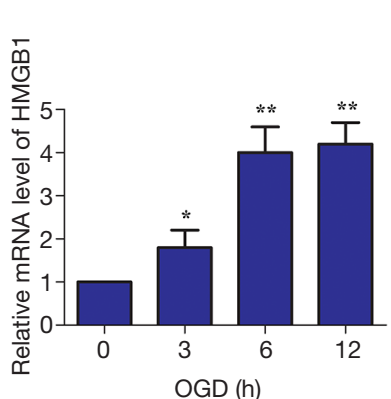

$\mathrm{D} \underset{\overline{\mathrm{O}}}{\overline{\mathrm{N}}}$

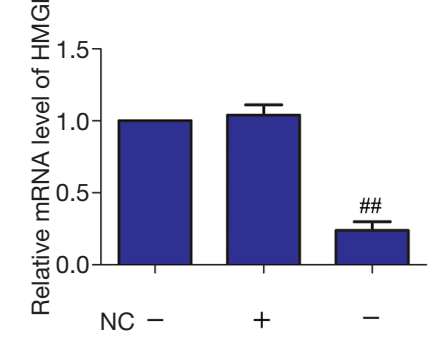

miR-103a-3p - $\quad-\quad+$
B
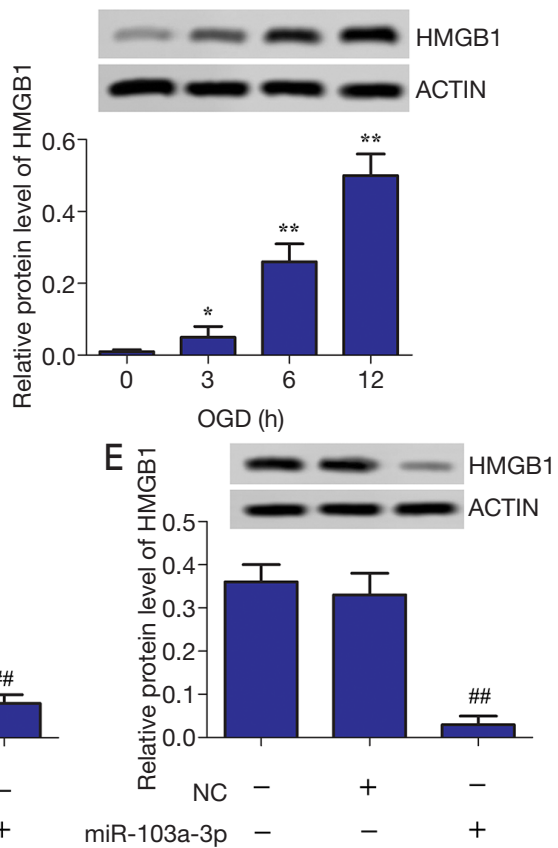

G

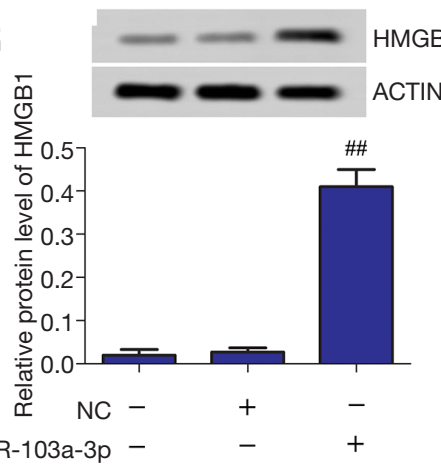

$\mathrm{H}$

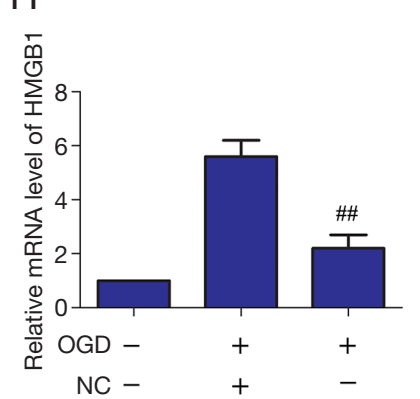
anti-miR-103a-3p -
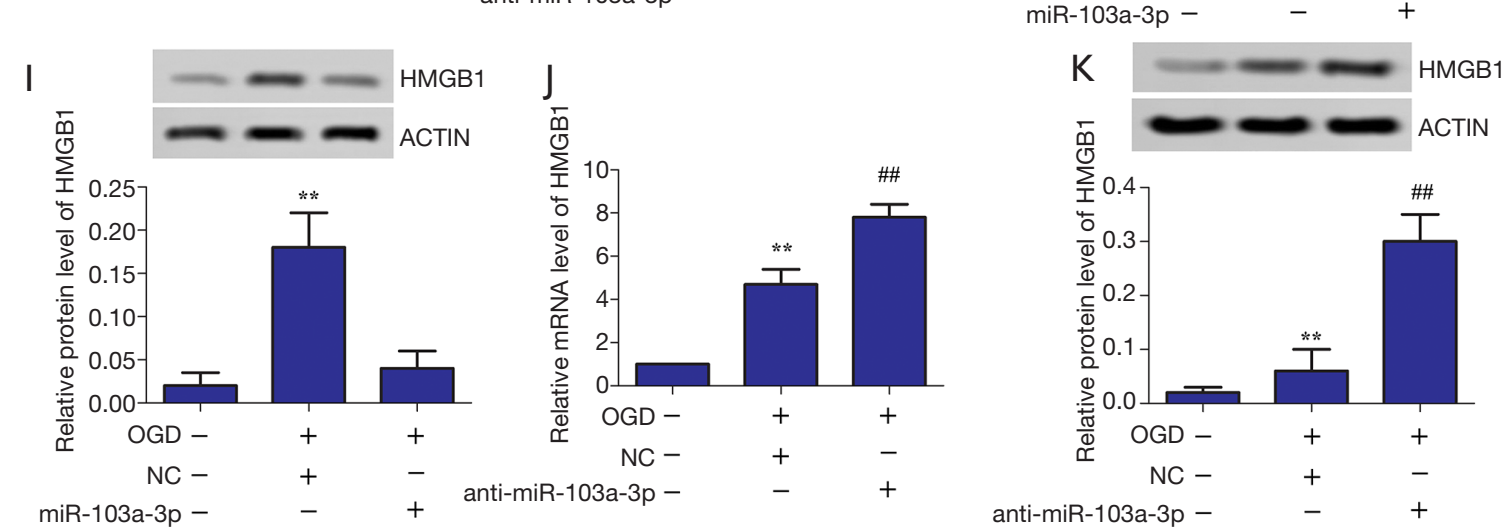

Figure 1 Influence of miR-103a-3p interference on HMGB1 expression in OGD- stimulated BV2 cells. (A,B) Effect of OGD treatment time on the expression of miR-103a-3p and HMGB1 in BV2 microglial cells. (C) miR-103a-3p mimic and anti-miR-103a-3p mimic were successfully constructed. (D,E,F,G) The effects of miR-103a-3p mimic and anti-miR-103a-3p mimic on HMGB1 expression in BV2 microglial cells, respectively. (H,I,G,K) Effects of miR-103a-3p mimic and anti-miR-103a-3p mimic on HMGB1 expression in OGDstimulated BV2 microglial cells, respectively $\left({ }^{*}, \mathrm{P}<0.05,{ }^{* *}, \mathrm{P}<0.01\right.$, versus the control group; ${ }^{* \#}, \mathrm{P}<0.01$ versus the miR-103a-3p NC group or the anti-miR-103a-3p NC group). HMGB1, high mobility group box 1; OGD, oxygen-glucose deprivation. 
A

Position 50-56 of HMGB1 3' UTR WT 5' ...UGAUAUUUUGgaUAUUGCUGCAU...

| || | | hsa-miR-103a-3p 3' AGUAUCGGGACAUGUUACGACGA HMGB1 3' UTR MUT 5 ' ...UGAUAUUUUGGAUAUCAGACUU...

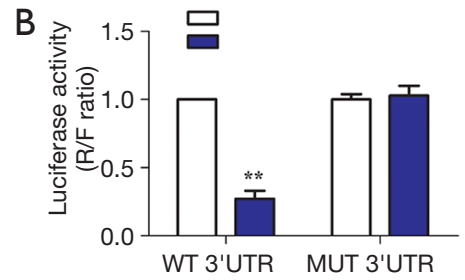

D
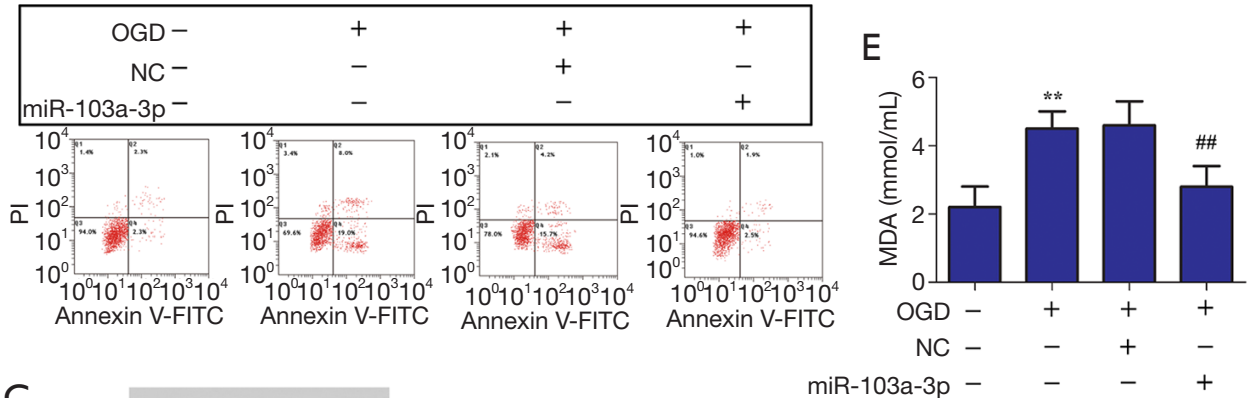

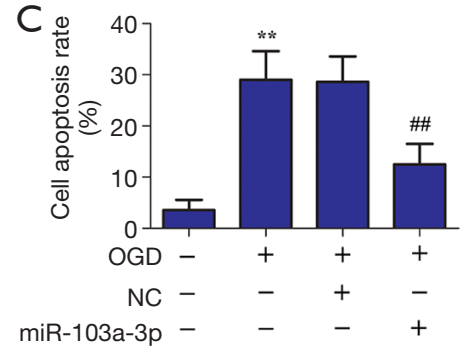

$\mathrm{F}$

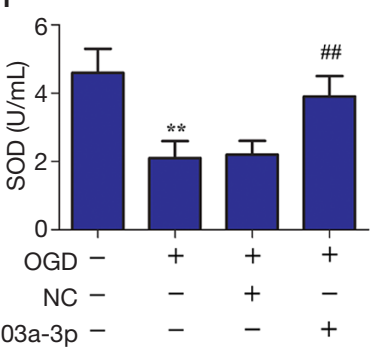

G

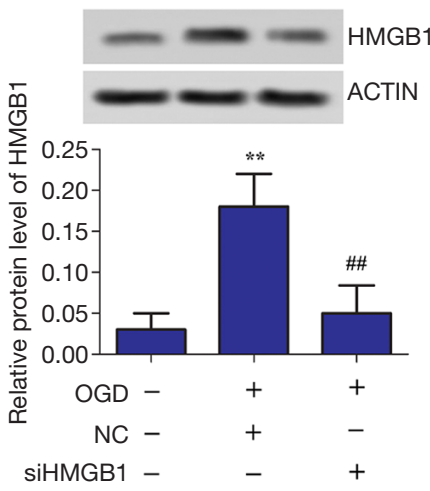

$\mathrm{H}$

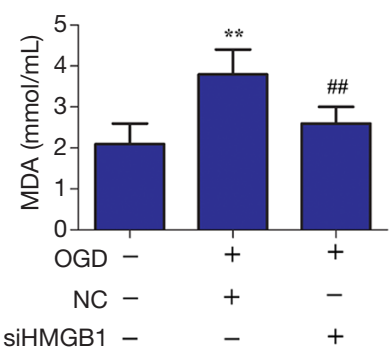

I

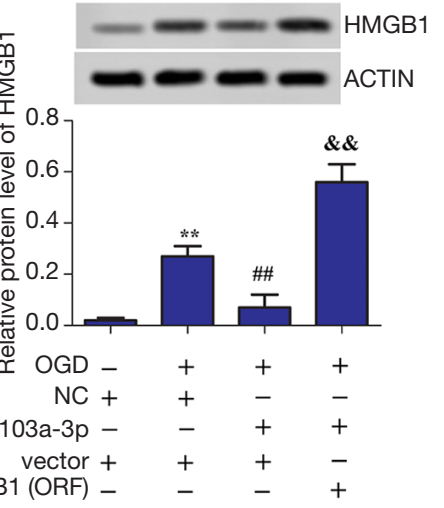

K
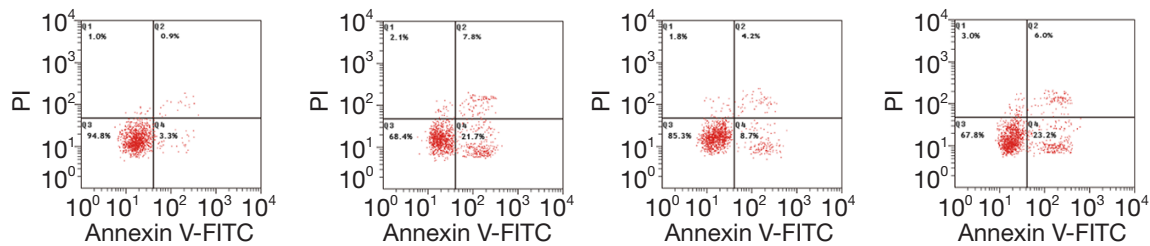

$10^{0} 10^{1} 10^{2} 10^{3} 10^{4}$
Annexin V-FITC
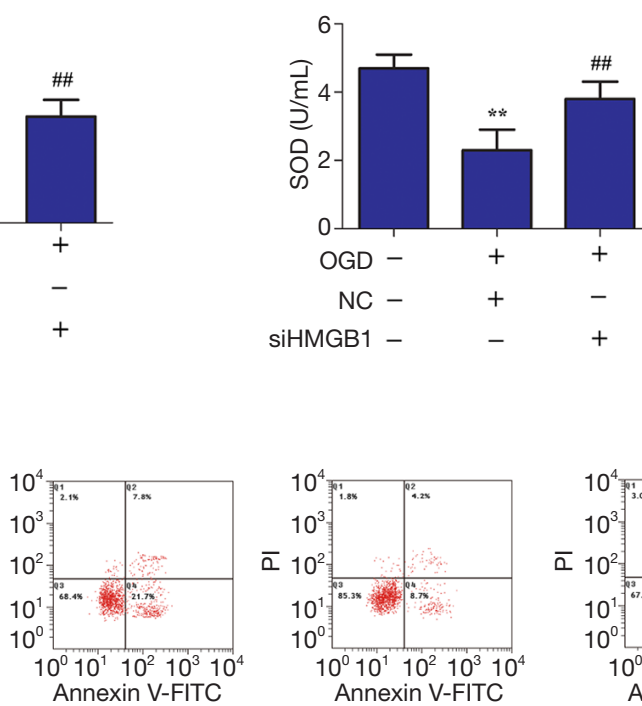

OGD -
$\mathrm{NC}+$
miR-103a-3p -
vector +
HMGB1 (ORF) -
Annexin V-FITC

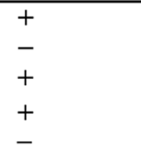
Annexin V-FITC

HMGB1 (ORF) $-\quad-\quad+\quad+$ 

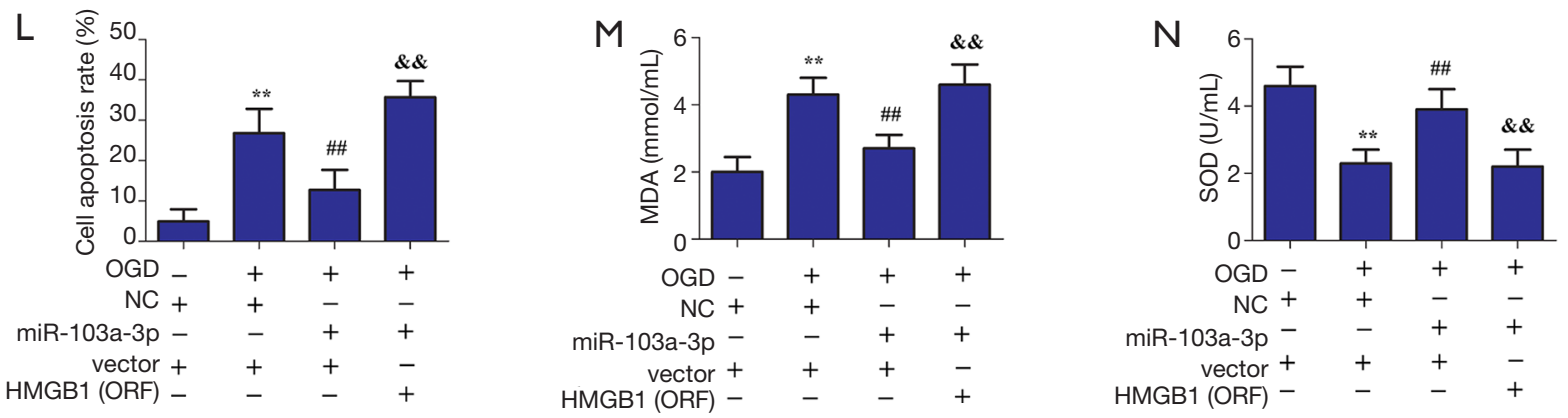

Figure 2 miR-103a-3p alleviated LPS-induced oxidative stress and apoptosis in BV2 glial cells by targeting HMGB1. (A) The results predicted by TargetScan search software. (B) The ratio of R/F obtained from the luciferase activity experiment. (C,D) Effects of miR-103a$3 p$ overexpression on the rate of cell apoptosis. (E,F) Effects of miR-103a-3p overexpression on MDA and SOD content. (G) Effects of HMGB1 interference on HMGB1 expression in OGD-stimulated BV2 microglial cells. (H,I) Effects of HMGB1 interference on MDA and SOD contents in OGD-stimulated BV2 microglial cells. (J) Effects of HMGB1 overexpression on HMGB1 expression in OGD-stimulated BV2 microglial cells. (K,L) Effects of miR-103a-3p and HMGB1 overexpression on the rate of cell apoptosis in OGD-stimulated BV2 microglial cells. (M,N) Effects of miR-103a-3p and HMGB1 overexpression on MDA and SOD content in OGD-stimulated BV2 microglial cells (** $\mathrm{P}<0.01$ versus the control group; ${ }^{\# \#}, \mathrm{P}<0.01$ versus the miR-103a-3p NC group; ${ }^{\& \&}, \mathrm{P}<0.01$ versus the miR-103a-3p mimic group). MDA, malondialdehyde; SOD, superoxide dismutase; HMGB1, high mobility group box 1; OGD, oxygen-glucose deprivation.

of HMGB1 3'-UTR-mut vector (Figure 2B). As shown in Figure 2C,D,E,F, overexpression of miR-103a-3p inhibited oxidative stress and apoptosis in OGD-treated BV2 cells. Further, siHMGB1 and HMGB1 (ORF) were successfully constructed (Figure 2G). Figure 2H,I show that interfering with HMGB1 suppressed oxidative stress in OGD-treated BV2 cells. As shown in Figure 27,K,L, M,N, HMGB1 overexpression promoted apoptosis and oxidative stress in OGD-treated BV2 cells. These results indicated that miR$103 a-3 p$ alleviates LPS-induced oxidative stress in OGDtreated BV2 glial cells by targeting HMGB1.

\section{Effect of miR-103a-3p on immune disorder in OGD- treated BV2 cells by targeting HMGB1}

Inflammation is well known to play a major role in mediating brain injury caused by cerebral ischemia. As shown in Figure 3A, miR-103a-3p overexpression decreased the content of IL- $1 \beta$ and TNF- $\alpha$, but enhanced the content of IL-4 and IL-10, in OGD-treated BV2 glial cells. As reflected in Figure 3B, interference with HMGB1 decreased the levels of TNF- $\alpha$ and IL- $1 \beta$, while enhancing the levels of IL-4 and IL-10. As shown in Figure 3C, OGD treatment increased the levels of IL-10, IL-4, IL-1 $\beta$, and TNF- $\alpha$ compared with the control group. Interestingly, the overexpression of miR-103a-3p decreased the levels of IL- $1 \beta$ and TNF- $\alpha$ but enhanced the levels of IL- 4 and IL-10 in OGD-treated BV2 glial cells. However, HMGB1 overexpression reversed these effects compared with the miR-103a-3p overexpression group. These findings suggested that miR-103a-3p alleviates immune disorder in OGD-treated BV2 cells by targeting HMGB.

\section{miR-103a-3p exerts protective effects on brain injury in a CI/R rat model}

$\mathrm{HE}$ and Nissl staining were used to detect brain injury in the rats. As shown in Figure 4A, compared with the sham group, the brains of the CI/R group rats showed significant injury. Compared with the CI/R group, miR-103a-3p NC had little effect on the brain injury, while miR-103a-3p mimics significantly reversed the injury. Compared with the sham group, the cerebral indexes, cerebral water content percentage, neurologic deficit score, and postural reflex score were all increased in the CI/R group; however, miR$103 a-3 p$ overexpression alleviated brain injury (Figure $4 B$ ). Interestingly, there was no significant difference between miR-103a-3p mimics group and nifedipine treated positive control group. These results indicated that miR-103a-3p exerted a protective effect against brain injury in a rat model of cerebral ischemia-reperfusion.

\section{miR-103a-3p alleviates oxidative stress, apoptosis and immune disorder in rats with CI/R by targeting HMGB1}

To further investigate the effects of miR-103a-3p on 

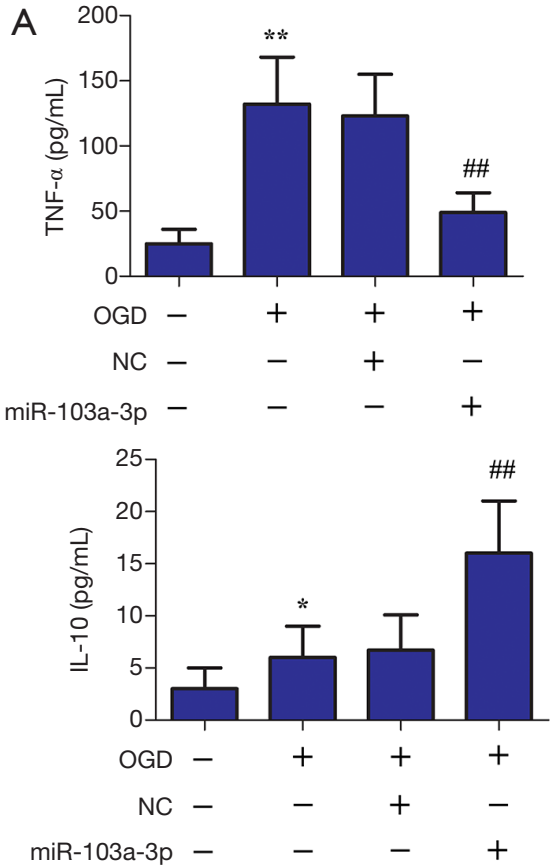

B

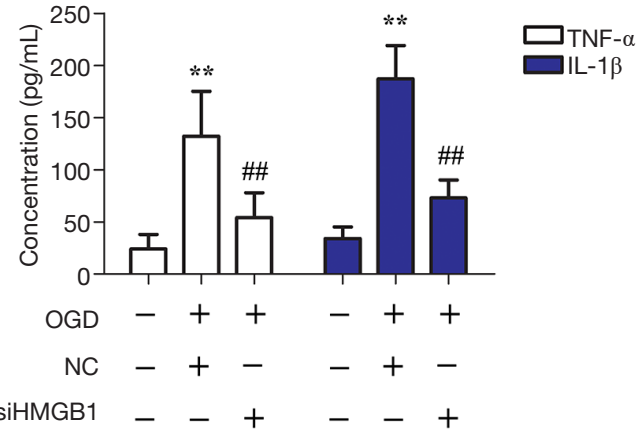

C

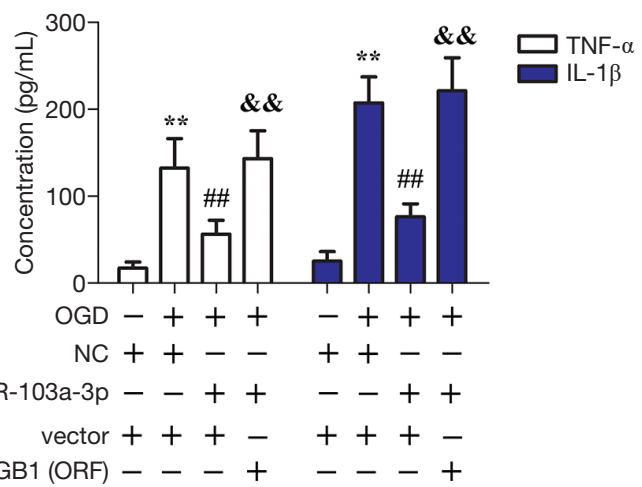

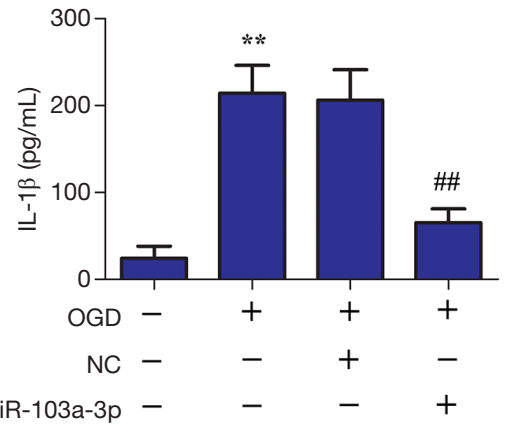
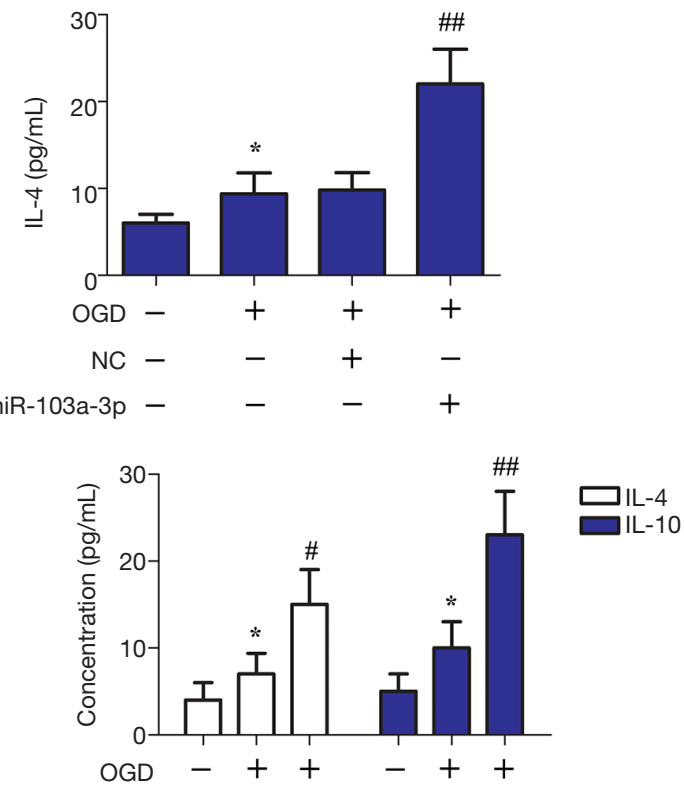

$$
\mathrm{NC}-+-+-
$$$$
\text { siHMGB1 }-\quad+\quad-\quad+
$$

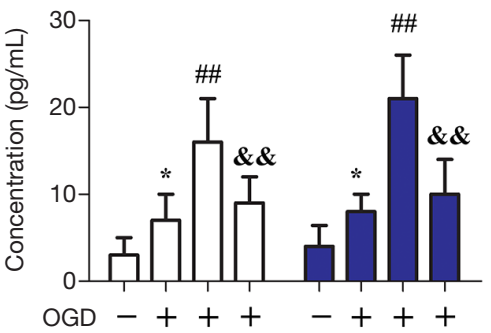

$\mathrm{NC}++--++-$

miR-103a-3p -++-++

vector +++-++

HMGB1 (ORF) ---+---+

Figure 3 Effect of miR-103a-3p on immune disorder in OGD-treated BV2 cells by targeting HMGB1. (A) Effects of miR-103a-3p overexpression on the levels of TNF- $\alpha$, IL-1 $\beta$, IL-4, and IL-10 expression in OGD-stimulated BV2 microglial cells. (B) Effects of HMGB1 interference on the levels of TNF- $\alpha$, IL-1, IL-4, and IL-10 expression in OGD-stimulated BV2 microglial cells. (C) Effects of miR-103a$3 p$ and HMGB1 overexpression on the levels of TNF- $\alpha$, IL-1 $\beta$, IL-4, and IL-10 expression in OGD-stimulated BV2 microglial cells (*, $\mathrm{P}<0.05 ;$ **, $\mathrm{P}<0.01$ versus the control group; ${ }^{\#}, \mathrm{P}<0.05$ versus the miR-103a-3p NC group; ${ }^{\# \#, ~} \mathrm{P}<0.01$ versus the miR-103a-3p NC group; \&\&, $\mathrm{P}<0.01$ versus the miR-103a-3p mimic group). OGD, oxygen-glucose deprivation; HMGB1, high mobility group box 1; TNF- $\alpha$, tumor necrosis factor-alpha; IL-1 $\beta$, interleukin-1beta; IL-4, interleukin-4; IL-10, interleukin-10. 
A Sham

$\mathrm{Cl} / \mathrm{R}$

$\mathrm{Cl} / \mathrm{R}+\mathrm{miR}-103 \mathrm{a}-3 p \mathrm{NC}$

$\mathrm{Cl} / \mathrm{R}+\mathrm{miR}-103 \mathrm{a}-3 \mathrm{p}$ mimics
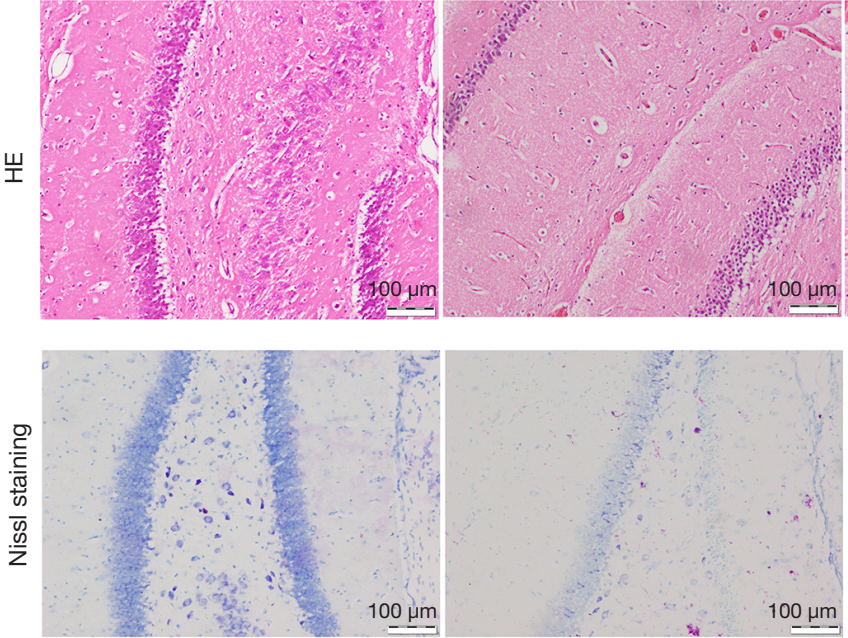

B
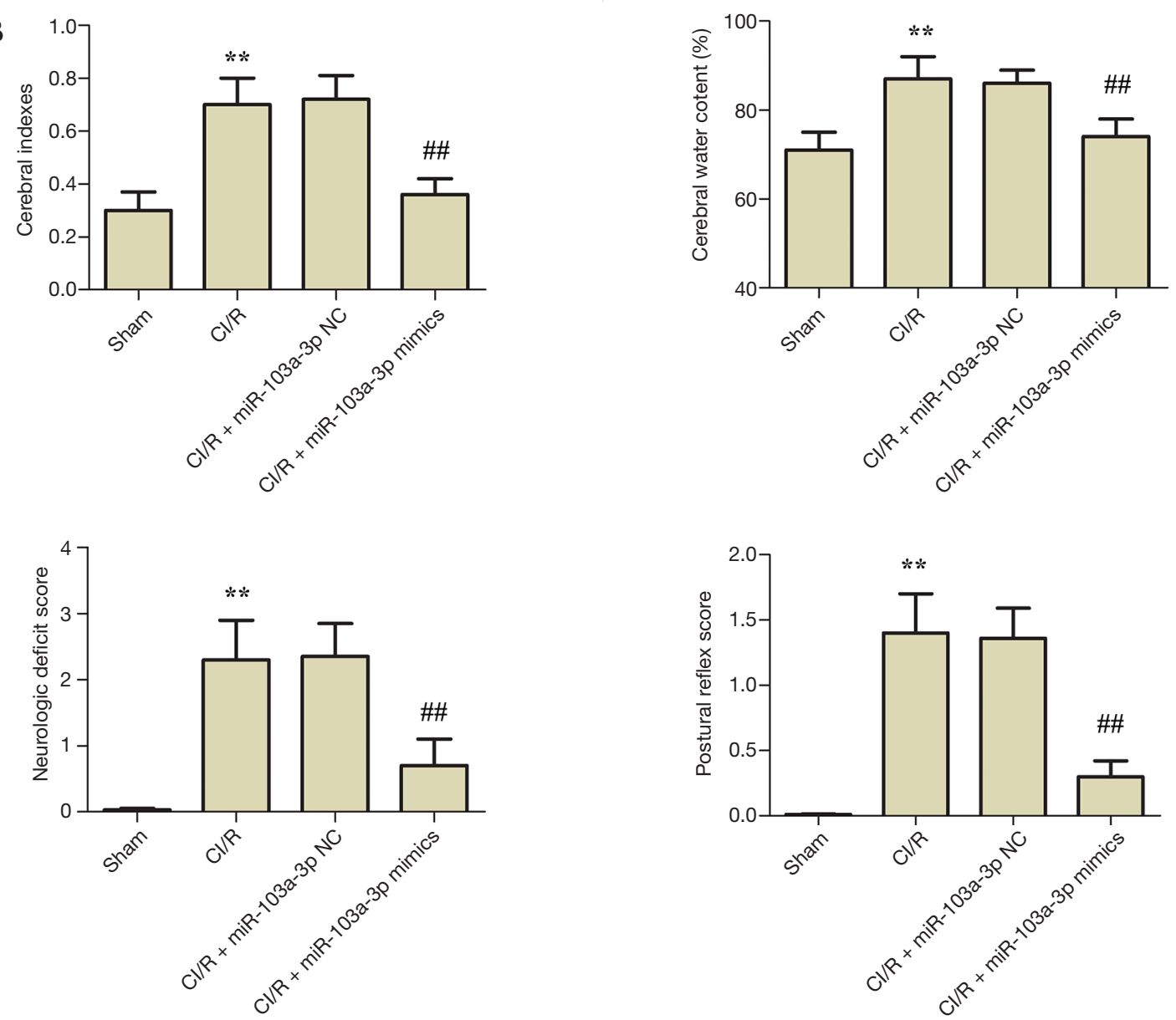

Figure 4 miR-103a-3p exerted protective effects against brain injury in a cerebral ischemia-reperfusion rat model. (A) Typical images of

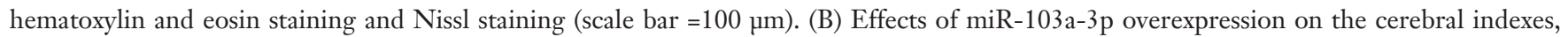
percentage of cerebral water content, neurological deficit score, and postural reflex score ${ }^{* *}, \mathrm{P}<0.01$ versus the control group; ${ }^{\# \#}, \mathrm{P}<0.01$ versus the $\mathrm{CI} / \mathrm{R}$ group). $\mathrm{CI} / \mathrm{R}$, cerebral ischemia-reperfusion injury; $\mathrm{H} \& \mathrm{E}$, hematoxylin and eosin. 

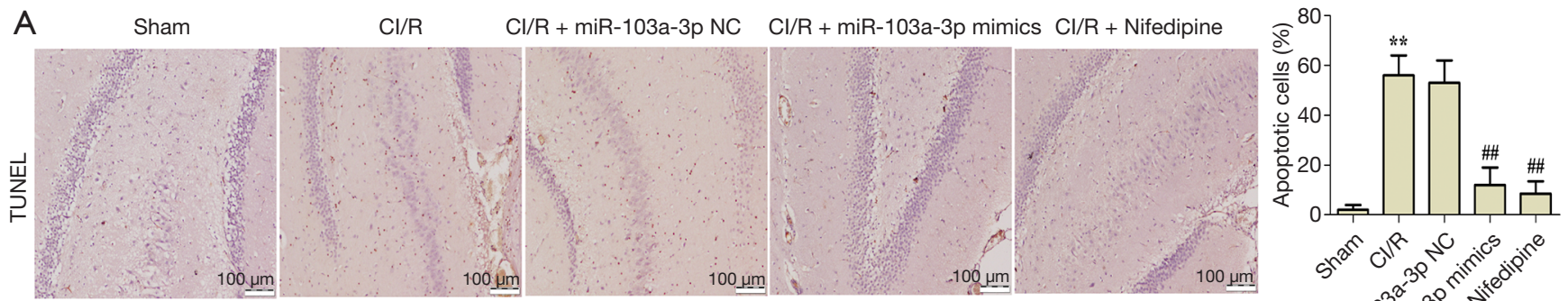

B
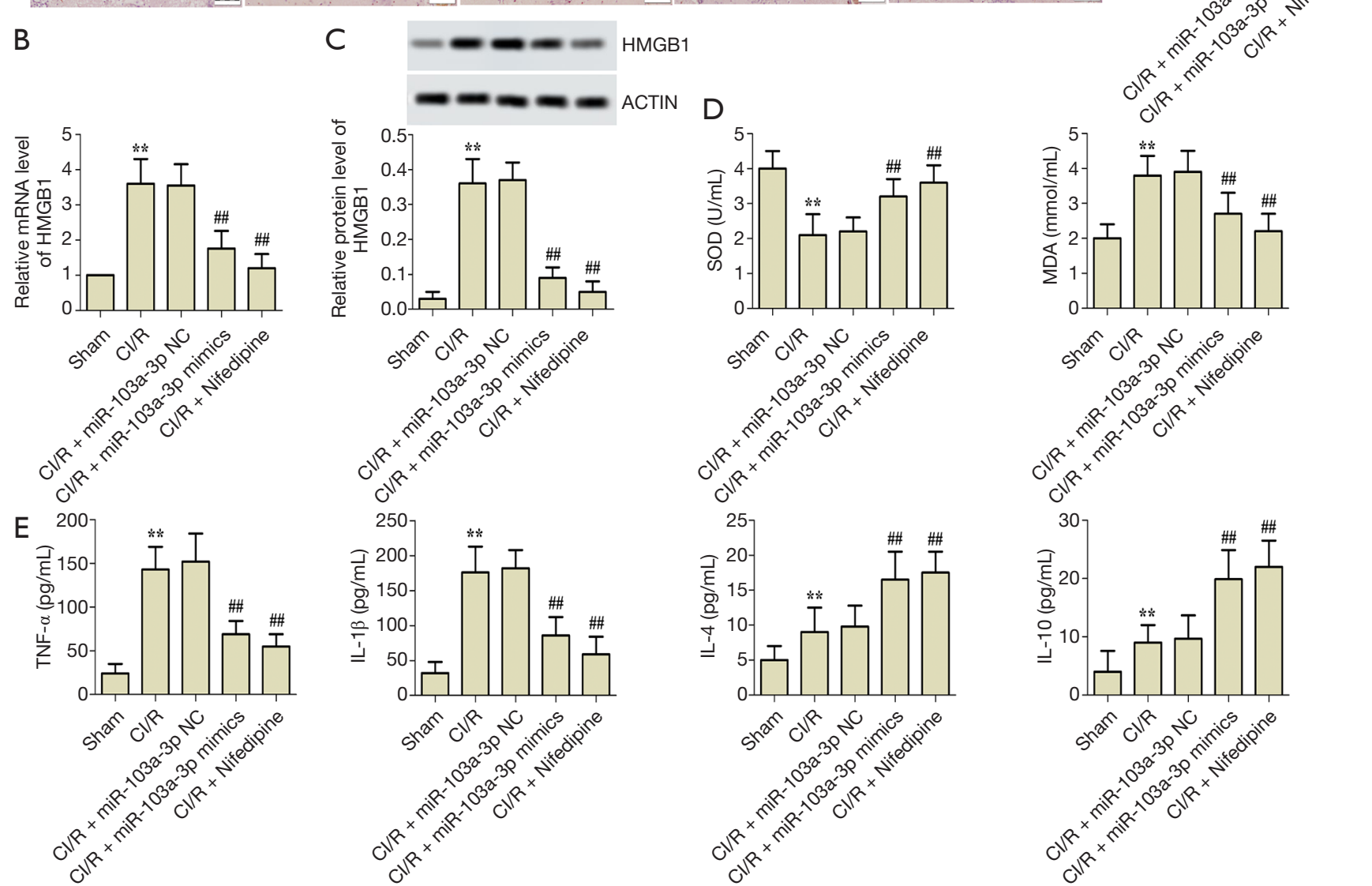

Figure 5 miR-103a-3p alleviated oxidative stress, apoptosis, and immune disorder in rats with CI/R by targeting HMGB1. (A) Typical images of TUNEL assay and the rate of cell apoptosis (scale bar $=100 \mu \mathrm{m}$ ). (B,C) Effects of miR-103a-3p overexpression on HMGB1 expression in vivo. (D) Effects of miR-103a-3p overexpression on MDA and SOD content in vivo. (E) Effects of miR-103a-3p overexpression on the levels of TNF- $\alpha$, IL-1 $\beta$, IL-4 and IL-10 expression in vivo (** $\mathrm{P}<0.01$ versus the control group; ${ }^{\# \#}, \mathrm{P}<0.01$ versus the CI/R group). $\mathrm{CI} / \mathrm{R}$, cerebral ischemia-reperfusion injury; HMGB1, high mobility group box 1; TUNEL, terminal deoxynucleotidyl transferase dUTP nick end labeling; MDA, malondialdehyde; SOD, superoxide dismutase; TNF- $\alpha$, tumor necrosis factor-alpha; IL-1 $\beta$, interleukin-1beta; IL4, interleukin-4; IL-10, interleukin-10.

$\mathrm{CI} / \mathrm{R}$ rats, brain tissue apoptosis, oxidative stress, and inflammatory factors were detected in vivo. According to the results of the TUNEL assay, compared with the sham group, the rate of cell apoptosis was significantly enhanced in the CI/R group; however, this rate was significantly decreased by miR-103a-3p overexpression (Figure $5 A$ ).
The levels of HMGB1 expression in brain tissue of the rats were detected by RT-PCR and western blot. As shown in Figure 5B,C, compared with the sham group, the expression of HMGB1 was significantly upregulated in the $\mathrm{CI} / \mathrm{R}$ group; however, compared with the CI/R group, miR-103a-3p overexpression downregulated HMGB 1 
expression significantly. As shown in Figure 5D, compared with the sham group, the SOD content was decreased and the MDA content was increased in the CI/R group. Interestingly, compared with the CI/R group, miR-103a-3p overexpression significantly increased the content of SOD and decreased the content of MDA. Furthermore, Figure $5 E$ shows that miR-103a-3p overexpression significantly reversed the abnormal expressions of TNF- $\alpha$, IL- $1 \beta$, IL4 , and IL-10 in the CI/R rat model. Of note, there was no significant difference between miR-103a-3p mimics group and nifedipine treated positive control group. These results suggested that miR-103a-3p alleviated oxidative stress, apoptosis, and immune disorder in rats with $\mathrm{CI} / \mathrm{R}$ by targeting HMGB1.

\section{Discussion}

Cerebral ischemia is the most common clinical feature of circulatory arrest. It results in delayed neuronal damage in vulnerable brain areas, such as the hippocampal CA1 region $(28,29)$. After cerebral ischemia, the recovery of the blood supply cannot restore brain function, and a more serious dysfunction problem develops, which is called $\mathrm{CI} / \mathrm{R}$. Despite the great progress that has been made in understanding the mechanism of cerebral ischemia/ reperfusion injury in the last decade, there is a lack of effective therapeutic targets. Therefore, it is necessary to find new drugs to treat cerebral ischemia. The results of our study demonstrated that overexpression of miR-103-3p or interference with HMGB1 attenuated oxidative stress and relieved immune disorder, and inhibited apoptosis in cells. We found that HMGB1 was negatively regulated by miR103-3p. Moreover, we identified that miR-103-3p alleviated $\mathrm{CI} / \mathrm{R}$ by targeting HMGB1 in vitro and in vivo. Our findings revealed a novel mechanism regulating cerebral ischemia.

$\mathrm{CI} / \mathrm{R}$ is understood to be a complex pathological process, which involves inflammation caused by white blood cell aggregation, oxidative stress, calcium overload due to increased intracellular $\mathrm{Ca}^{2+}$ flow and reduced discharge $(30,31)$. An increasing amount of evidence has showed that cerebral ischemia triggers inflammatory response and leads to microglia activation, which generates more cytotoxic substances including TNF- $\alpha$, IL- $1 \beta$, and other pro-inflammatory mediators, resulting in further neuronal damage $(32,33)$. To mimic the $\mathrm{CI} / \mathrm{R}$ conditions in vitro in our experiment, BV2 microglial cells underwent OGD/ $\mathrm{R}$ treatment. In this study, we found that OGD treatment time was inversely proportional to the expression of miR-
$103-3 p$ and in direct proportion to the expression of HMGB1 in BV2 microglial cells. Interestingly, TargetScan search software predicted HMGB1 as a target of miR-1033 p. Moreover, we found that overexpression of HMGB1 enhanced the expression levels of IL- $1 \beta$ and TNF- $\alpha$, but decreased the expression levels of IL-4 and IL-10. These results are consistent with those of previous studies. Fiuza et al. reported that human microvascular endothelial cells stimulated with HMGB1 in vitro exhibited increased expression of proinflammatory cytokine TNF- $\alpha$ (17). Chen et al. reported that a high glucose level induced the secretion of HMGB1 and inflammatory cytokines in mesangial cells, while interference with HMGB1 significantly reduced the levels of glucose-induced inflammatory cytokines via regulating the NF- $\mathrm{\kappa B}$ signaling pathway (34). HMGB1 is well known as an important mediator of organ damage with strong proinflammatory effects. HMGB1 is generally localized in the nucleus; however, under inflammatory stimuli, it translocates to the cytoplasm and is released into the extracellular milieu. Outside cells, HMGB1 usually binds to advanced glycation end products (RAGE), tolllike receptor 2 (TLR2), and TLR4, and then activates several signaling pathways, including the NF- $\kappa \mathrm{B}$ signaling pathway (35). The inhibitory effect of miR-103-3p on HMGB1-induced inflammation may also be related to the NF- $\mathrm{KB}$ signaling pathway.

Mitochondria are the regulatory centers of energy metabolism and oxidative stress. Oxidative stress, which is related to mitochondrial damage, is another important pathological process in CI/R. miR-103 expression is reported to mediate the downregulation of $\mathrm{Bcl}-2 / \mathrm{E} 1 \mathrm{~B}-$ $19 \mathrm{kDa}$-interacting protein 3 (BNIP3), and further protects cells by inhibiting oxidative stress (9). HMGB1 plays crucial roles in the regulation of cellular response to intracellular or extracellular stress. Although the mechanisms that contribute to the biology of HMGB1 are still under investigation, oxidative stress appears to be a major regulator of HMGB1 translocation and release, as well as inflammation and cell death activity (36). Therefore, we inferred that targeting HMGB1 with antioxidant compounds may be an attractive therapeutic strategy for treating inflammatory diseases such as CI/R necrosis and apoptosis. In our study, miR-103-3p overexpression and interference with HMGB1 decreased the content of MDA, but increased the content of SOD; meanwhile, HMGB1 overexpression had the opposite effect. We also found that the overexpression of miR-103-3p and HMGB1 interference inhibited apoptosis, while HMGB1 overexpression had 
the opposite effect. These results suggest that miR-103a$3 \mathrm{p}$ alleviates oxidative stress and apoptosis in and rats with $\mathrm{CI} / \mathrm{R}$ injury by targeting HMGB1. CI/R injury stimulates secondary damage. We also found that miR-103-3p overexpression effectively alleviated brain tissue damage and regulated the related indicators, such as the cerebral indexes, percentage of cerebral water content, neurological deficit score, and postural reflex score. These findings were consistent with those of some earlier studies. Bai et al. found that circular RNA DLGAP4 improves the outcomes of ischemic stroke patients by targeting miR-143 to ameliorate endothelial-mesenchymal transition associated with the integrity of the blood-brain barrier (37). Also, while miR103 has been proved to be involved in brain disease $(38,39)$, the effect of mir-103a-3p on cerebral ischemia-reperfusion has not been reported.

In this study, we aimed to investigate whether miR$103 \mathrm{a}-3 \mathrm{p}$ alleviates $\mathrm{CI} / \mathrm{R}$ in vivo and vitro and explored the relevant mechanisms. Our results suggested that HMGB1 can be upregulated significantly in OGD-stimulated BV2 microglial cells and in a CI/R rat model. We also found that the HMGB1 gene is a target of miR-103a-3p. Furthermore, miR-103a-3p overexpression could silence HMGB1 and reversed abnormal phenomena induced by HMGB1 upregulation in vitro and in vivo. Thus, we speculated that miR-103a-3p alleviates oxidative stress, immune disorder, and apoptosis in OGD-stimulated BV2 microglial cells and rats with CI/R injury via targeting HMGB1. Our experiments were limited by the small number of samples, and further research is needed to establish a deeper understanding of the mechanism underlying the effect of miR-103a-3p on CI/R injury.

\section{Acknowledgments}

Funding: None.

\section{Footnote}

Reporting Checklist: The authors have completed the ARRIVE reporting checklist. Available at http://dx.doi. org/10.21037/atm-20-5856

Data Sharing Statement: Available at http://dx.doi. org/10.21037/atm-20-5856

Conflicts of Interest: All authors have completed the ICMJE uniform disclosure form (available at http://dx.doi. org/10.21037/atm-20-5856). The authors have no conflicts of interest to declare.

Ethical Statement: The authors are accountable for all aspects of the work in ensuring that questions related to the accuracy or integrity of any part of the work are appropriately investigated and resolved. All animal experiments were approved by the Ethics Committee of the Institutional Animal Care and Use Committee of Xinxiang Central Hospital (Henan, China) (No. XXZXYY-2019093) and conducted in line with the Guide for the Care and Use of Laboratory Animals (2011).

Open Access Statement: This is an Open Access article distributed in accordance with the Creative Commons Attribution-NonCommercial-NoDerivs 4.0 International License (CC BY-NC-ND 4.0), which permits the noncommercial replication and distribution of the article with the strict proviso that no changes or edits are made and the original work is properly cited (including links to both the formal publication through the relevant DOI and the license). See: https://creativecommons.org/licenses/by-nc-nd/4.0/.

\section{References}

1. Johnston SC, Mendis S, Mathers CD. Global variation in stroke burden and mortality: estimates from monitoring, surveillance, and modelling. Lancet Neurol 2009;8:345-54.

2. Margaill I, Plotkine M, Lerouet D. Antioxidant strategies in the treatment of stroke. Free Radic Biol Med 2005;39:429-43.

3. Chan PH. Role of oxidants in ischemic brain damage. Stroke 1996;27:1124-9.

4. Bernhagen J. "Remote" myokine protects from pulmonary ischemia/reperfusion injury by a surprising "proximal" control mechanism. Ann Transl Med 2018;6:275.

5. Hembrom AA, Srivastava S, Garg I, et al. MicroRNAs in venous thrombo-embolism. Clin Chim Acta 2020;504:66-72.

6. Fagan AM, Perrin RJ. Upcoming candidate cerebrospinal fluid biomarkers of Alzheimer's disease. Biomark Med 2012;6:455-76.

7. Lages E, Ipas H, Guttin A, et al. MicroRNAs: molecular features and role in cancer. Front Biosci (Landmark Ed) 2012;17:2508-40.

8. Di Y, Lei Y, Yu F, et al. MicroRNAs expression and function in cerebral ischemia reperfusion injury. J Mol Neurosci 2014;53:242-50. 
9. Xu MC, Gao XF, Ruan C, et al. miR-103 Regulates Oxidative Stress by Targeting the BCL2/Adenovirus E1B $19 \mathrm{kDa}$ Interacting Protein 3 in HUVECs. Oxid Med Cell Longev 2015;2015:489647.

10. Yang H, Wang H, Shu Y, et al. miR-103 Promotes Neurite Outgrowth and Suppresses Cells Apoptosis by Targeting Prostaglandin-Endoperoxide Synthase 2 in Cellular Models of Alzheimer's Disease. Front Cell Neurosci 2018;12:91.

11. Li G, Chen T, Zhu Y, et al. MiR-103 alleviates autophagy and apoptosis by regulating SOX2 in LPS-injured PC12 cells and SCI rats. Iran J Basic Med Sci 2018;21:292-300.

12. Xi Z, Qiao Y, Wang J, et al. Gastrodin relieves inflammation injury induced by lipopolysaccharides in MRC-5 cells by up-regulation of miR-103. J Cell Mol Med 2020;24:1451-9.

13. Kang C, Kang M, Han Y, et al. 6-Gingerols (6G) reduces hypoxia-induced PC-12 cells apoptosis and autophagy through regulation of miR-103/BNIP3. Artif Cells Nanomed Biotechnol 2019;47:1653-61.

14. Lu B, Wang C, Wang M, et al. Molecular mechanism and therapeutic modulation of high mobility group box 1 release and action: an updated review. Expert Rev Clin Immunol 2014;10:713-27.

15. Tian J, Avalos AM, Mao SY, et al. Toll-like receptor 9-dependent activation by DNA-containing immune complexes is mediated by HMGB1 and RAGE. Nat Immunol 2007;8:487-96.

16. Yu M, Wang H, Ding A, et al. HMGB1 signals through toll-like receptor (TLR) 4 and TLR2. Shock 2006;26:174-9.

17. Fiuza C, Bustin M, Talwar S, et al. Inflammationpromoting activity of HMGB1 on human microvascular endothelial cells. Blood 2003;101:2652-60.

18. Yan S, Fang C, Cao L, et al. Protective effect of glycyrrhizic acid on cerebral ischemia/reperfusion injury via inhibiting HMGB1-mediated TLR4/NF- $\mathrm{KB}$ pathway. Biotechnol Appl Biochem 2019;66:1024-30.

19. Wang C, Jiang J, Zhang X, et al. Inhibiting HMGB1 Reduces Cerebral Ischemia Reperfusion Injury in Diabetic Mice. Inflammation 2016;39:1862-70.

20. Li XQ, Chen FS, Tan WF, et al. Elevated microRNA129-5p level ameliorates neuroinflammation and bloodspinal cord barrier damage after ischemia-reperfusion by inhibiting HMGB1 and the TLR3-cytokine pathway. J Neuroinflammation 2017;14:205.

21. Mo ZT, Li WN, Zhai YR, et al. The effects of icariin on the expression of HIF-1 $\alpha$, HSP-60 and HSP-70 in PC12 cells suffered from oxygen-glucose deprivation-induced injury. Pharm Biol 2017;55:848-52.

22. Liu X, Zhu X, Chen M, et al. Resveratrol protects PC12 cells against OGD/ R-induced apoptosis via the mitochondrial-mediated signaling pathway. Acta Biochim Biophys Sin (Shanghai) 2016;48:342-53.

23. El-Abhar HS, Shaalan M, Barakat M, et al. Effect of melatonin and nifedipine on some antioxidant enzymes and different energy fuels in the blood and brain of global ischemic rats. J Pineal Res 2002;33:87-94.

24. Magnain C, Augustinack JC, Reuter M, et al. Blockface histology with optical coherence tomography: a comparison with Nissl staining. Neuroimage 2014;84:524-33.

25. Zhu JY, Yang X, Chen Y, et al. Curcumin Suppresses Lung Cancer Stem Cells via Inhibiting Wnt/ $\beta$-catenin and Sonic Hedgehog Pathways. Phytother Res 2017;31:680-8.

26. Longa EZ, Weinstein PR, Carlson S, et al. Reversible middle cerebral artery occlusion without craniectomy in rats. Stroke 1989;20:84-91.

27. Yin XM, Luo Y, Cao G, et al. Bid-mediated mitochondrial pathway is critical to ischemic neuronal apoptosis and focal cerebral ischemia. J Biol Chem 2002;277:42074-81.

28. Kim MJ, Cho JH, Cho JH, et al. Impact of hyperthermia before and during ischemia-reperfusion on neuronal damage and gliosis in the gerbil hippocampus induced by transient cerebral ischemia. J Neurol Sci 2015;348:101-10.

29. Kong Z, Shen Q, Jiang J, et al. Wogonin improves functional neuroprotection for acute cerebral ischemia in rats by promoting angiogenesis via TGF- $\beta 1$. Ann Transl Med 2019;7:639.

30. Khoshnam SE, Winlow W, Farzaneh $M$, et al. Pathogenic mechanisms following ischemic stroke. Neurol Sci 2017;38:1167-86.

31. Rekuviene E, Ivanoviene L, Borutaite V, et al. Rotenone decreases ischemia-induced injury by inhibiting mitochondrial permeability transition in mature brains. Neurosci Lett 2017;653:45-50.

32. Iadecola C, Alexander M. Cerebral ischemia and inflammation. Curr Opin Neurol 2001;14:89-94.

33. Kaushal V, Schlichter LC. Mechanisms of microgliamediated neurotoxicity in a new model of the stroke penumbra. J Neurosci 2008;28:2221-30.

34. Chen Y, Qiao F, Zhao Y, et al. HMGB1 is activated in type 2 diabetes mellitus patients and in mesangial cells in response to high glucose. Int J Clin Exp Pathol 2015;8:6683-91.

35. Herzog C, Lorenz A, Gillmann HJ, et al. 
Thrombomodulin's lectin-like domain reduces myocardial damage by interfering with HMGB1-mediated TLR2 signalling. Cardiovasc Res 2014;101:400-10.

36. Yu Y, Tang D, Kang R. Oxidative stress-mediated HMGB1 biology. Front Physiol 2015;6:93.

37. Bai Y, Zhang Y, Han B, et al. Circular RNA DLGAP4 Ameliorates Ischemic Stroke Outcomes by Targeting miR143 to Regulate Endothelial-Mesenchymal Transition Associated with Blood-Brain Barrier Integrity. J Neurosci 2018;38:32-50.

38. Shi FP, Wang XH, Zhang HX, et al. MiR-103 regulates

Cite this article as: $\mathrm{Li} \mathrm{J}, \mathrm{He} W$, Wang $\mathrm{Y}$, Zhao J, Zhao X. miR-103a-3p alleviates oxidative stress, apoptosis, and immune disorder in oxygen-glucose deprivation-treated BV2 microglial cells and rats with cerebral ischemia-reperfusion injury by targeting high mobility group box 1 . Ann Transl Med 2020;8(20):1296. doi: 10.21037/atm-20-5856 the angiogenesis of ischemic stroke rats by targeting vascular endothelial growth factor (VEGF). Iran J Basic Med Sci 2018;21:318-24.

39. Vinciguerra A, Formisano L, Cerullo P, et al. MicroRNA-103-1 selectively downregulates brain NCX1 and its inhibition by anti-miRNA ameliorates stroke damage and neurological deficits. Mol Ther 2014;22:1829-38.

(English Language Editor: J. Reynolds) 